\title{
Simultaneous Quantitation of 13 Active Components in SimiaoYong'an Decoction Using High-Performance Liquid Chromatography with Diode Array Detection
}

\author{
Liang Zheng ${ }^{1}$, Mengyue Wang ${ }^{1}$, Zhong Chen ${ }^{2}$, Jincai Hou ${ }^{3}$ and Xiaobo Li ${ }^{1 *}$ \\ ${ }^{1}$ School of Pharmacy, Shanghai Jiao Tong University, Shanghai 200240, China \\ ${ }^{2}$ Shineway Pharmaceutical Group Co., Ltd., Shijiazhuang 051430, China \\ ${ }^{3}$ Jing-Jin-Ji Joint Innovation Pharmaceutical (Beijing) Co., Ltd., Beijing 100083, China
}

Received: 03 March 2018; accepted: 08 April 2018

\begin{abstract}
SimiaoYong'an decoction, a traditional Chinese medicine formula consisting of four herbs, has been widely used for the treatment of gangrene disease. However, its clinical application is restricted due to the lack of an effective quality control method that covers the main active compounds in the formula. In this study, a high-performance liquid chromatography with diode-array detection (HPLC-DAD) method was established for the simultaneous determination of 13 active compounds including harpagide, neochlorogenic acid, chlorogenic acid, cryptochlorogenic acid, ferulic acid, isochlorogenic acid $\mathrm{B}$, isochlorogenic acid $\mathrm{A}$, isochlorogenic acid $\mathrm{C}$, angoroside $\mathrm{C}$, harpagoside, cinnamic acid, glycyrrhizic acid, and ligustilide. Separation of these compounds was achieved using a Kromasil $100-5-\mathrm{C}_{18}$ column with a gradient elution program consisting of acetonitrile and $0.4 \%$ phosphoric acid solution. The specificity, linearity, precision, repeatability, and accuracy tests were implemented to validate the method. The validated method was successfully applied for determination of 13 components from several finished batches of SimiaoYong'an decoction. The results demonstrated that the established method was accurate, reliable, and could be used as a suitable quality control method for the quantification of SimiaoYong'an decoction.
\end{abstract}

Keywords: Active components, quantitative analysis, SimiaoYong'an decoction, traditional Chinese medicine

\section{Introduction}

SimiaoYong'an decoction (SYD) is a famous Chinese herbal formula firstly recorded in Hua Tuo Shen Yi Mi Zhuan in Han Dynasty and then listed in Yan Fang Xin Bian by Bao Xiang'ao in Qing Dynasty [1]. SYD is composed of Lonicerae japonicae flos (dried flower bud or opening flower of Lonicera japonica Thunb.), Scrophulariae radix (dried root of Scrophularia ningpoensis Hemsl.), Angelicae sinensis radix (dried root of Angelica sinensis [Oliv.] Diels.), and Glycyrrhizae radix et rhizoma (dried root and rhizome of Glycyrrhiza uralensis Fisch.). It has been used for approximately a thousand years to treat gangrene, clear heat, remove toxicity, and alleviate pain $[2,3]$. Modern pharmacological studies have shown that SYD has broad clinical applications for treating thromboangiitis obliterans [4], atherosclerosis [5], diabetic foot [6], and gouty arthritis [3].

The efficacy of Traditional Chinese medicines (TCMs), especially TCM preparations, depends on multiple constituents, but not a single compound. The present quality control method of quantifying one or two major components recorded in Chinese Pharmacopoeia can hardly ensure the clinical effectiveness. Therefore, quantitative determination of multiple active components is considered as the most important and direct method for quality control of TCM preparations [7-9]. To date, few studies have reported on the quantitative analysis methods of main active components for the quality control of SYD, which restricts the clinical application of this preparation.

Recently, 14 compounds of SYD were identified in rat serum, of which 9 were in the original form, such as chlorogenic acid, isochlorogenic acid $\mathrm{C}$, harpagide, harpagoside,

\footnotetext{
*Author for correspondence: School of Pharmacy, Shanghai Jiao Tong University, Shanghai 200240, China; xbli@sjtu.edu.cn; +86-21-34204806; +86-21$3420-4804$
}

and angoroside $\mathrm{C}[10]$. In addition, a method of ultra-performance liquid chromatography-tandem mass spectrometry (UPLCMS/MS) was established for the simultaneous determination of seven ingredients of SYD extract in rat plasma for its pharmacodynamic study, and the marker compounds were selected from Lonicerae japonicae flos, Scrophulariae radix, and Glycyrrhizae radix et rhizoma, while the constituents from Angelicae sinensis radix were not taken into account [1]. According to the compatibility theory of Chinese medicine of SYD, Lonicerae japonicae flos, the monarch herb which plays the most critical role in the formula, was confirmed to contain organic acids, flavonoids, iridoid glycosides, and saponins; particularly organic acids such as chlorogenic acid, neochlorogenic acid, cryptochlorogenic acid, isochlorogenic acid $\mathrm{A}$, isochlorogenic acid $\mathrm{B}$, and isochlorogenic acid $\mathrm{C}$ make a great contribution to its anti-inflammatory, antibacterial, and antioxidant effects [11-14]. Similarly, Scrophulariae radix as the minister herb, which enhance the efficacy of the monarch herb, is mainly composed of iridoids (such as harpagide and harpagoside), phenylethanoid glycosides (such as angoroside C), and organic acids (such as cinnamic acid) [15-17]. On the other hand, the assistant herb, Angelicae sinensis radix, supported the monarch and minister herbs to reach target positions and exert their effects. It mainly consists of organic acids (such as ferulic acid) and volatile oils (such as ligustilide), which exert many pharmacological activities such as anti-thrombosis, anti-inflammatory, and antimicrobial [18-20]. Glycyrrhizae radix et rhizoma, used as supplementary herb to coordinate with other herbs, is mainly composed of flavonoids and saponins, and glycyrrhizic acid is the typical constituents with extensive pharmacological effects as well as high content in its water extract $[21,22]$. Based on the above, thirteen compounds, which have certain pharmacological effects and relatively high contents for quantitative analysis, were selected for the quality

This is an open-access article distributed under the terms of the Creative Commons Attribution-NonCommercial 4.0 International License (https://creativecommons.org/licenses/by-nc/4.0/), which permits unrestricted use, distribution, and reproduction in any medium for non-commercial purposes, provided the original author and source are credited, a link to the CC License is provided, and changes - if any - are indicated. 
control of SYD: neochlorogenic acid, chlorogenic acid, cryptochlorogenic acid, isochlorogenic acid $\mathrm{B}$, isochlorogenic acid A and isochlorogenic acid $\mathrm{C}$ from Lonicerae japonicae flos, harpagide, angoroside $\mathrm{C}$, harpagoside and cinnamic acid from Scrophulariae radix, ferulic acid and ligustilide from Angelicae sinensis radix, and glycyrrhizic acid from Glycyrrhizae radix et rhizoma. The chemical structures of these compounds are shown in Figure 1. In this study, a method of high-performance liquid chromatography with diode-array detection (HPLCDAD) was established for simultaneous determination of multiple active compounds for the first time, which will contribute to the establishment of a comprehensive quality control method of SYD.

\section{Experimental}

2.1 Chemicals, Reagents, and Materials. The four crude herbs of several different batches, Lonicerae japonicae flos,
Scrophulariae radix, Angelicae sinensis radix, and Glycyrrhizae radix et rhizoma, were purchased from Nanjing Haiyuan Chinese Medicine Tablet Co., Ltd. (Nanjing, China) and Sichuan Mianyang Pharmaceutical Co., Ltd., Taiji Group (Mianyang, China). Their botanical origins were identified by the corresponding author, and voucher specimens were deposited at the School of Pharmacy, Shanghai Jiao Tong University. The reference standards of neochlorogenic acid, chlorogenic acid, cryptochlorogenic acid, ferulic acid, isochlorogenic acid B, isochlorogenic acid $\mathrm{A}$, isochlorogenic acid $\mathrm{C}$, angoroside $\mathrm{C}$, harpagoside, cinnamic acid, glycyrrhizic acid, and ligustilide (purity, >98\%) were purchased from Sichuan Weikeqi Biological Co., Ltd. (Chengdu, China). Reference standard of harpagide (purity, 96.8\%) was purchased from the National Institutes for Food and Drug Control (Beijing, China). Acetonitrile, methanol, and phosphoric acid of HPLC grade were obtained from ANPEL Laboratory Technologies Inc. (Shanghai, China). Ultrapure water was prepared by a Milli-Q system (Millipore, MA, USA).

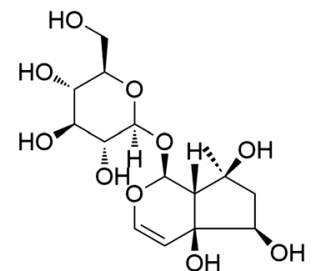

(1)<smiles>O=C(/C=C/c1ccc(O)c(O)c1)OC1C[C@](O)(C(=O)O)C[C@H](O)[C@H]1O</smiles>

(2)<smiles>O=C(/C=C/c1ccc(O)c(O)c1)OC1C[C@](O)(C(=O)O)C[C@H](O)[C@H]1O</smiles>

(3)<smiles>COc1cc(/C=C/C(=O)O)ccc1O</smiles><smiles>O=C(/C=C/c1ccc(O)c(O)c1)O[C@H]1CC(O)(C(=O)O)C[C@@H](OC(=O)/C=C/c2ccc(O)c(O)c2)[C@@H]1O</smiles>

(7)

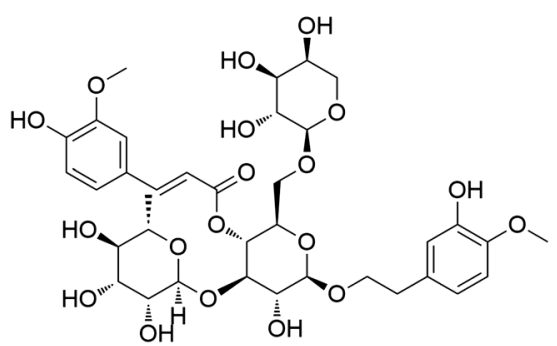

(9)<smiles>O=C(/C=C/c1ccc(O)c(O)c1)OC1[C@@H](O)CC(O)(C(=O)O)C[C@@H]1OC(=O)/C=C/c1ccc(O)c(O)c1</smiles>

(8)<smiles>CC1(O[C@H]2OC=CC3(O)C(O)CC(C)(OC(=O)/C=C/c4ccccc4)[C@H]23)O[C@H](CO)[C@@H](O)[C@H](O)C1O</smiles>

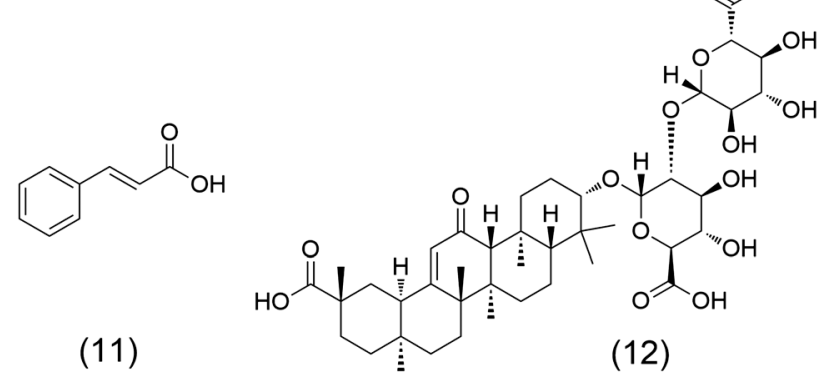<smiles>CCC/C=C1\OC(=O)C2=C3C=CCCC3=C21</smiles>

Figure 1. Chemical structures of the thirteen marker constituents in SYD. The numbers (1)-(13) are as follows: (1) harpagide, (2) neochlorogenic acid, (3) chlorogenic acid, (4) cryptochlorogenic acid, (5) ferulic acid, (6) isochlorogenic acid B, (7) isochlorogenic acid A, (8) isochlorogenic acid C, (9) angoroside C, (10) harpagoside, (11) cinnamic acid, (12) glycyrrhizic acid, and (13) ligustilide 
2.2 Chromatographic Conditions and Instrumentation. Analyses were performed on an Agilent 1200 HPLC system, equipped with a quaternary solvent delivery system, an on-line degasser, an autosampler, a column temperature controller, and a DAD detector. A Kromasil 100-5-C18 $(250 \mathrm{~mm} \times 4.6 \mathrm{~mm}$, $5 \mu \mathrm{m}$ ) column was employed during the experiment with a flow rate of $1.0 \mathrm{~mL} / \mathrm{min}$. The column temperature was maintained at $38^{\circ} \mathrm{C}$, and the injection volume was $10 \mu \mathrm{L}$. The mobile phase was composed of water (with $0.4 \%$ phosphoric acid) (A) and acetonitrile (B) with the following gradient elution: 0-3 min, 95\% A; 3-18 min, 95\%-89\% A; 18-45 min, 89\%-80\% A; 45-55 min, 80-67\% A; 55-60 min, 67\%-50\% A; 60-70 min, $50 \%-0 \% \mathrm{~A}$, and reequilibration for $5 \mathrm{~min}$. The wavelength was set at $210 \mathrm{~nm}$ for harpagide; $254 \mathrm{~nm}$ for glycyrrhizic acid; $278 \mathrm{~nm}$ for chlorogenic acid, harpagoside, and cinnamic acid; and $327 \mathrm{~nm}$ for neochlorogenic acid, cryptochlorogenic acid, ferulic acid, isochlorogenic acid $\mathrm{B}$, isochlorogenic acid $\mathrm{A}$, isochlorogenic acid $\mathrm{C}$, angoroside $\mathrm{C}$, and ligustilide.

2.3 Preparation of Sample Solutions. According to the original composition of SYD, the four herbs including Lonicerae japonicae flos (11.25 g), Scrophulariae radix (11.25 g), Angelicae sinensis radix (7.5 g), and Glycyrrhizae radix et rhizoma (3.75 g) were weighed accurately, mixed, and then immersed in 10 fold volume of distilled water for $30 \mathrm{~min}$. Subsequently, the mixture was decocted for $40 \mathrm{~min}$ in a casserole. The extracted solution was centrifuged at $4000 \mathrm{rpm}$ for $15 \mathrm{~min}$, and the supernatant was concentrated under reduced pressure to a final volume of $100 \mathrm{~mL}$.

For HPLC analysis, $1 \mathrm{~mL}$ methanol was added to equal volume of SYD solution in a centrifuge tube. After being vortexed for $3 \mathrm{~min}$, the mixture was centrifuged at $12,000 \mathrm{rpm}$ for $10 \mathrm{~min}$. The supernatant was then transferred into a $2 \mathrm{~mL}$ dark brown volumetric flask and bring to volume by $50 \%$ methanol solution. The prepared solution was filtered through a $0.22 \mu \mathrm{m}$ membrane filter before injection into the HPLC-DAD system for analysis.

2.4 Preparation of Standard Solutions. Stock standard solution of the 13 reference compounds was prepared by dissolving them with $50 \%$ methanol in a $10 \mathrm{~mL}$ dark brown volumetric flask and then diluted to a series of proper concentrations of mixed standard solutions. The concentrations of the 13 reference compounds were the following: harpagide: $438.50,350.80,175.40,87.70,43.85,21.93$, and $10.96 \mu \mathrm{g} / \mathrm{mL}$; neochlorogenic acid: $153.62,122.90,61.45,30.72,15.36,7.68$, and $3.84 \mu \mathrm{g} / \mathrm{mL}$; chlorogenic acid: 1416.23, 1132.99, 566.49, $283.25,141.62,70.81$, and $35.41 \mu \mathrm{g} / \mathrm{mL}$; cryptochlorogenic acid: 254.64, 203.71, 101.85, 50.93, 25.46, 12.73, and $6.37 \mu \mathrm{g} / \mathrm{mL}$; ferulic acid: 110.90, 88.72, 44.36, 22.18, 11.09, 5.55, and $2.77 \mu \mathrm{g} / \mathrm{mL}$; isochlorogenic acid B: 219.71, 175.77, 87.89, 43.94, 21.97, 10.99, and $5.49 \mu \mathrm{g} / \mathrm{mL}$; isochlorogenic acid A: 461.26, 369.01, 184.50, 92.25, 46.13, 23.06, and $11.53 \mu \mathrm{g} / \mathrm{mL}$; isochlorogenic acid C: 324.02, 259.22, 129.61, 64.80, 32.40, 16.20, and $8.10 \mu \mathrm{g} / \mathrm{mL}$; angoroside C: $154.98,123.99,61.99$, $31.00,15.50,7.75$, and $3.87 \mu \mathrm{g} / \mathrm{mL}$; harpagoside: $120.74,96.59$, $48.30,24.15,12.07,6.04$, and $3.02 \mu \mathrm{g} / \mathrm{mL}$; cinnamic acid: $167.58,134.06,67.03,33.52,16.76,8.38$, and $4.19 \mu \mathrm{g} / \mathrm{mL}$; glycyrrhizic acid: 396.22, 316.98, 158.49, 79.24, 39.62, 19.81, and $9.91 \mu \mathrm{g} / \mathrm{mL}$; and ligustilide: 134.94, 107.95, 53.98, 26.99, $13.49,6.75$, and $3.37 \mu \mathrm{g} / \mathrm{mL}$. All the solutions were stored at $4{ }^{\circ} \mathrm{C}$ and filtered through a $0.22 \mu \mathrm{m}$ membrane filter before injection.

2.5 Preparation of Negative Control Samples of SYD. The three negative control samples of SYD were prepared by removal of one herb from the prescriptions, respectively. The rest of herbs were accurately weighed according to the prescription of SYD and prepared by the same procedure described in Section 2.3.
2.6 Method Validation. The analytical method was validated for specificity, linearity, limit of detection (LOD), limit of quantification (LOQ), precision, repeatability, stability, and accuracy in accordance with Chinese Pharmacopoeia (ChP) 2015 [23]. The specificity of the method was evaluated by comparing the chromatograms of standard solution, sample solution, and negative control samples. The standard curves for the linearity assay were based on plotting the peak areas versus the corresponding concentrations of each analyte. Linearity test solutions for the assay method were prepared from stock solution, as detailed in Section 2.4. To determine the LODs and LOQs, the lowest diluted solutions for calibration use were further diluted to a series of concentrations. They were then analyzed until the signal-to-noise $(\mathrm{S} / \mathrm{N})$ ratio for each analyte was about 3 for LOD and 10 for LOQ. The intra-day and inter-day precision were assessed by analyzing six replicates of
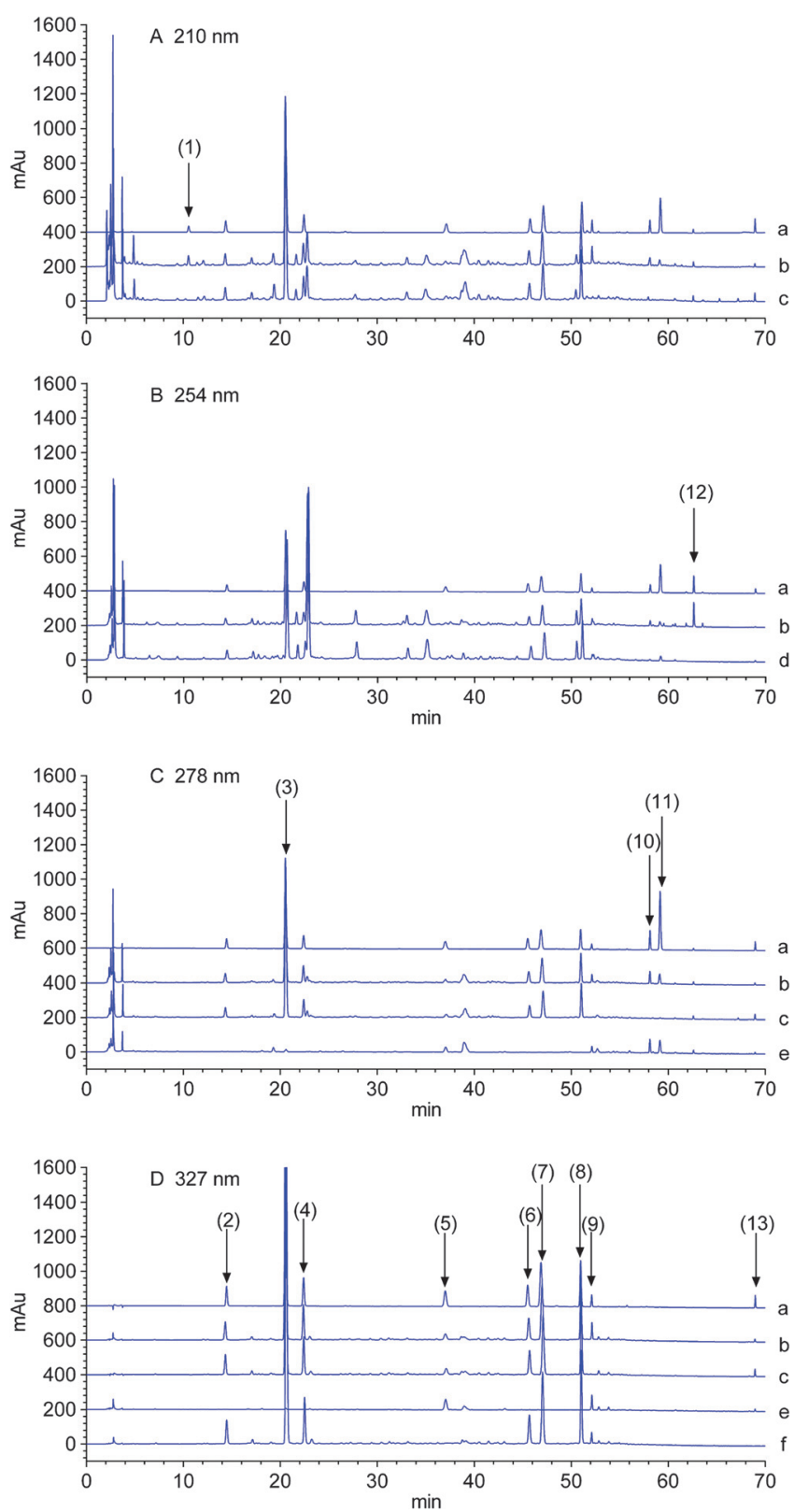

Figure 2. Typical HPLC-DAD chromatograms for determination of the 13 active compounds. (a) Standard mixture; (b) SYD test sample; (c) test sample without Scrophulariae radix; (d) test sample without Glycyrrhizae radix et rhizoma; (e) test sample without Lonicerae japonicae flos; (f) test sample without Angelicae sinensis radix. The entry numbers refer to compounds as cited in Figure 1 
a mixed standard solution during a single day and by duplicating the experiments on three successive days. To confirm the repeatability, six sample solutions in parallel were prepared according to method of Section 2.3. and analyzed with the method mentioned above. The relative standard deviation (RSD) was calculated as a measurement of precision and repeatability. Stability was tested with SYD sample solution at room temperature and analyzed at $0,2,4,8,12,24$, and $48 \mathrm{~h}$ after preparation. In the recovery test, samples were prepared at three concentration levels in triplicate by spiking known amount of the mixed standard solutions into the SYD solutions, and then processed and analyzed according to the described procedures. The average recovery (\%) of 13 compounds at three levels was calculated according to the following equation:

$$
\begin{aligned}
\operatorname{Recovery}(\%)= & (\text { detected amount }- \text { original amount }) \\
& / \text { spiked amount } \times 100 \% .
\end{aligned}
$$

\section{Results and Discussion}

3.1 Optimization of Chromatographic Conditions. The types of chromatographic column, mobile phase compositions, gradient elution program, column temperature, and detection wavelengths were all tested to optimize the separation conditions for HPLC analysis. Different columns including Kromasil 100-5- $\mathrm{C}_{18}(250 \mathrm{~mm} \times 4.6 \mathrm{~mm}, 5 \mu \mathrm{m})$, Agilent $\mathrm{XDB}^{-\mathrm{C}_{18}}(250 \mathrm{~mm} \times 4.6 \mathrm{~mm}, 5 \mu \mathrm{m})$, and Shimadzu shimpack $\mathrm{C}_{18}(250 \mathrm{~mm} \times 4.6 \mathrm{~mm}, 5 \mu \mathrm{m})$ were tested, and the results showed that Kromasil $100-5-\mathrm{C}_{18}$ column was the most suitable one, since it gave better peak shapes and a good separation of selected analytes. The mobile phases with different acids (formic acid and phosphoric acid) and acidity were investigated, and the combination of water (with $0.4 \%$ phosphoric acid) and acetonitrile for mobile phase was the best for separation. Column temperature and flow rate were also the critical influencing factors on the multicomponent separation, and the separation could be improved with a column temperature of $38{ }^{\circ} \mathrm{C}$ and a flow rate of $1 \mathrm{~mL} / \mathrm{min}$. Furthermore, different gradient elution programs were also optimized. We tried to shorten the analysis time and simplify the gradient elution systems, but peaks for isochlorogenic acid $\mathrm{C}$ and angoroside $\mathrm{C}$, as well as harpagoside and cinnamic acid, have not been completely separated except for the current gradient elution program mentioned above. The ultraviolet (UV) wavelength of the DAD for the quantification of each component was selected at $210 \mathrm{~nm}$ (harpagide), $254 \mathrm{~nm}$ (glycyrrhizic acid), $278 \mathrm{~nm}$ (chlorogenic acid, harpagoside, and cinnamic acid), and $327 \mathrm{~nm}$ (neochlorogenic acid, cryptochlorogenic acid, ferulic acid, isochlorogenic acid $\mathrm{B}$, isochlorogenic acid $\mathrm{A}$, isochlorogenic acid $\mathrm{C}$, angoroside
$\mathrm{C}$, and ligustilide) based on retention time and UV spectra compared with those of the reference standards, and each of the 13 compounds had the higher UV absorption and could be determined without interference from other components at the selected UV wavelength.

3.2 Method Validation. The analytical method was validated for specificity, linearity, LOD, LOQ, precision, repeatability, stability, and accuracy.

3.2.1 Specificity. Representative chromatograms of the mixed standard solution, SYD sample, and negative control samples are shown in Figure 2. The chromatographic peaks of the 13 components were identified by comparing the HPLC retention time and UV spectra with those of reference compounds. The retention times of the harpagide, neochlorogenic acid, chlorogenic acid, cryptochlorogenic acid, ferulic acid, isochlorogenic acid $\mathrm{B}$, isochlorogenic acid $\mathrm{A}$, isochlorogenic acid $\mathrm{C}$, angoroside $\mathrm{C}$, harpagoside, cinnamic acid, glycyrrhizic acid, and ligustilide were observed at 10.50, 14.28, 20.52, 22.38, $37.06,45.70,47.07,51.08,52.20,58.17,59.17,62.63$, and $68.96 \mathrm{~min}$, respectively. In addition, no interfering peak in the negative control samples was observed under the established chromatographic conditions, which further confirmed the specificity of this method.

3.2.2 Linearity, LOD, and $L O Q$. The linear regression equation, correlation coefficient $\left(R^{2}\right)$, linear range, LODs, and LOQs are presented in Table 1. Calibration curves of the 13 analytes showed good linearity, with $R^{2}$ values of $\geq 0.9998$ in the seven tested concentration ranges. The LODs and LOQs of the 13 analytes were $0.28-2.74$ and $0.92-9.91 \mu \mathrm{g} / \mathrm{mL}$, respectively.

3.2.3 Precision, Repeatability, and Stability. The results of the intra-day and inter-day precision, repeatability, and stability are summarized in Table 2. RSD values were used to evaluate the variations. The results showed that the values of intra- and inter-day precision were in the range of $0.22 \%-0.43 \%$ and

\begin{tabular}{|c|c|c|c|c|}
\hline \multirow[t]{2}{*}{ Compounds } & \multicolumn{2}{|c|}{ Precision (RSD\%) } & \multirow{2}{*}{$\begin{array}{l}\text { Repeatability } \\
\text { (RSD\%) }\end{array}$} & \multirow{2}{*}{$\begin{array}{l}\text { Stability } \\
\text { (RSD\%) }\end{array}$} \\
\hline & $\overline{\text { Intra-day }}$ & $\overline{\text { Inter-day }}$ & & \\
\hline Harpagide & 0.33 & 0.93 & 1.00 & 0.76 \\
\hline Neochlorogenic acid & 0.33 & 0.41 & 0.48 & 0.47 \\
\hline Chlorogenic acid & 0.25 & 0.33 & 0.40 & 0.16 \\
\hline Cryptochlorogenic acid & 0.22 & 0.71 & 0.42 & 0.14 \\
\hline Ferulic acid & 0.33 & 0.54 & 0.42 & 0.74 \\
\hline Isochlorogenic acid B & 0.36 & 0.36 & 0.43 & 0.33 \\
\hline Isochlorogenic acid $\mathrm{A}$ & 0.39 & 0.37 & 0.43 & 0.19 \\
\hline Isochlorogenic acid $\mathrm{C}$ & 0.32 & 0.35 & 0.47 & 0.25 \\
\hline Angoroside C & 0.43 & 0.38 & 0.45 & 0.76 \\
\hline Harpagoside & 0.27 & 0.30 & 0.49 & 0.34 \\
\hline Cinnamic acid & 0.29 & 0.29 & 0.29 & 0.21 \\
\hline Glycyrrhizic acid & 0.41 & 0.34 & 0.43 & 0.18 \\
\hline Ligustilide & 0.42 & 0.53 & 0.61 & 0.56 \\
\hline
\end{tabular}

Table 2. Precision, repeatability, and stability of 13 compounds in SYD

\begin{tabular}{|c|c|c|c|c|c|}
\hline Compounds & Regression equation & $R^{2}$ & Linear range $(\mu \mathrm{g} / \mathrm{mL})$ & LOD $(\mu \mathrm{g} / \mathrm{mL})$ & $\overline{L O Q}(\mu \mathrm{g} / \mathrm{mL})$ \\
\hline Harpagide & $y=3.695 x+7.0046$ & 0.9998 & $10.96-438.50$ & 2.74 & 6.85 \\
\hline Neochlorogenic acid & $y=37.477 x+17.061$ & 0.9999 & $3.84-153.62$ & 0.32 & 0.96 \\
\hline Chlorogenic acid & $y=13.935 x+84.033$ & 0.9998 & $35.41-1416.23$ & 0.89 & 2.53 \\
\hline Ferulic acid & $y=67.728 x-2.1599$ & 0.9999 & $2.77-110.90$ & 0.28 & 0.92 \\
\hline Isochlorogenic acid B & $y=35.64 x+4.1952$ & 0.9999 & $5.49-219.71$ & 0.39 & 0.92 \\
\hline Isochlorogenic acid $\mathrm{A}$ & $y=39.279 x+21.961$ & 0.9999 & $11.53-461.26$ & 0.64 & 1.92 \\
\hline Isochlorogenic acid $\mathrm{C}$ & $y=39.754 x+13.611$ & 0.9999 & $8.10-324.02$ & 0.68 & 2.03 \\
\hline Harpagoside & $y=30.962 x+13.778$ & 0.9998 & $3.02-120.74$ & 0.75 & 3.02 \\
\hline Cinnamic acid & $y=100.57 x+82.294$ & 0.9998 & $4.19-167.58$ & 0.42 & 1.40 \\
\hline Glycyrrhizic acid & $y=6.1048 x+9.0114$ & 0.9999 & $9.91-396.22$ & 2.48 & 9.91 \\
\hline Ligustilide & $y=14.324 x+7.6034$ & 0.9999 & $3.37-134.94$ & 0.56 & 1.69 \\
\hline
\end{tabular}

Table 1. Regression equation, correlation coefficient $\left(R^{2}\right)$, linear range, LODs, and LOQs of the 13 compounds in SYD 
Table 3. Recovery of 13 compounds in SYD $(n=3)$

\begin{tabular}{|c|c|c|c|c|c|}
\hline Compounds & $\begin{array}{l}\text { Original } \\
(\mu \mathrm{g} / \mathrm{mL})\end{array}$ & $\begin{array}{c}\text { Spiked } \\
(\mu \mathrm{g} / \mathrm{mL})\end{array}$ & $\begin{array}{l}\text { Detected } \\
(\mu \mathrm{g} / \mathrm{mL})\end{array}$ & $\begin{array}{c}\text { Recovery } \\
(\%)\end{array}$ & $\begin{array}{l}\text { RSD } \\
(\%)\end{array}$ \\
\hline \multirow[t]{3}{*}{ Harpagide } & 73.08 & 33.59 & 107.55 & 102.61 & 0.81 \\
\hline & & 67.18 & 138.27 & 97.03 & 0.57 \\
\hline & & 100.77 & 169.23 & 95.41 & 0.3 \\
\hline \multirow[t]{3}{*}{ Neochlorogenic acid } & 15.66 & 8.97 & 24.89 & 102.90 & 1.02 \\
\hline & & 17.94 & 33.42 & 98.98 & 0.52 \\
\hline & & 26.91 & 42.23 & 98.74 & 0.23 \\
\hline \multirow[t]{3}{*}{ Chlorogenic acid } & 288.25 & 144.79 & 439.83 & 104.69 & 0.65 \\
\hline & & 289.57 & 575.68 & 99.26 & 0 \\
\hline & & 434.36 & 717.44 & 98.81 & 0.22 \\
\hline \multirow[t]{3}{*}{ Cryptochlorogenic acid } & 31.86 & 14.37 & 46.75 & 103.65 & 0.8 \\
\hline & & 28.73 & 59.96 & 97.82 & 0 \\
\hline & & 43.10 & 73.86 & 97.47 & 0.0 \\
\hline \multirow[t]{3}{*}{ Ferulic acid } & 4.63 & 2.25 & 6.92 & 101.36 & 0. \\
\hline & & 4.51 & 9.06 & 98.15 & \\
\hline & & 6.76 & 11.35 & 99.32 & 0.4 \\
\hline \multirow[t]{3}{*}{ Isochlorogenic acid B } & 23.65 & 15.14 & 39.11 & 102.14 & 2. \\
\hline & & 30.28 & 53.25 & 97.98 & \\
\hline & & 45.43 & 68.82 & 99.43 & 0.2 \\
\hline \multirow[t]{3}{*}{ Isochlorogenic acid A } & 57.65 & 31.60 & 89.09 & 99.49 & 1.3 \\
\hline & & 63.20 & 118.42 & 96.15 & 0. \\
\hline & & 94.80 & 149.85 & 97.25 & 0.2 \\
\hline \multirow[t]{3}{*}{ Isochlorogenic acid $\mathrm{C}$} & 45.31 & 22.49 & 68.29 & 102.14 & 1.3 \\
\hline & & 44.99 & 89.39 & 97.98 & \\
\hline & & 67.48 & 112.04 & 98.89 & 0. \\
\hline \multirow[t]{3}{*}{ Angoroside C } & 21.34 & 13.54 & 35.47 & 104.39 & 0. \\
\hline & & 27.07 & 47.48 & 96.55 & 0. \\
\hline & & 40.61 & 60.06 & 95.35 & 0. \\
\hline \multirow[t]{3}{*}{ Harpagoside } & 7.35 & 4.95 & 12.51 & 104.29 & 1. \\
\hline & & 9.90 & 17.37 & 101.30 & \\
\hline & & 14.85 & 22.49 & 102.00 & 0. \\
\hline \multirow[t]{3}{*}{ Cinnamic acid } & 2.44 & 2.67 & 4.95 & 94.14 & 0. \\
\hline & & 5.34 & 7.73 & 99.20 & \\
\hline & & 8.00 & 10.59 & 101.83 & 0.2 \\
\hline \multirow[t]{3}{*}{ Glycyrrhizic acid } & 60.67 & 31.52 & 93.06 & 102.76 & 0.8 \\
\hline & & 63.05 & 122.44 & 97.97 & \\
\hline & & 94.57 & 153.81 & 98.49 & 0. \\
\hline \multirow[t]{3}{*}{ Ligustilide } & 2.76 & 2.48 & 5.13 & 95.65 & \\
\hline & & 4.95 & 7.79 & 101.59 & 0. \\
\hline & & 7.43 & 10.65 & 106.17 & 0.4 \\
\hline
\end{tabular}

$0.29 \%-0.93 \%$, respectively. The RSD values for the repeatability assessment of each analyte were $0.29 \%-1.00 \%$. The RSD values of the stability test were all less than $0.76 \%$, indicating that the sample solution was stable within $48 \mathrm{~h}$.

3.2.4 Accuracy. The accuracy of this method was assessed by a recovery test. The average recovery of the 13 compounds was in the range of $94.14 \%-106.17 \%$, and the RSD values were below $2.46 \%$. The results of the recovery test are summarized in Table 3.

3.3 Quantitative Analysis of SYD Samples. Each herb of six different batches was collected and randomly combined to prepare six batches of SYDs in accordance with the method described in Section 2.3. Thirteen marker compounds of SYD solutions were then determined by simultaneously applying the validated method above. The results are summarized in Table 4. Among the 13 components, chlorogenic acid was the most abundant compound (7.20-10.90 mg/g) followed by glycyrrhizic acid (3.23-7.62 $\mathrm{mg} / \mathrm{g})$, and ligustilide had the lowest amount $(0.06-0.17 \mathrm{mg} / \mathrm{g})$. Not only that, the average contents of 13 marker compounds were different in triplicates of 6 batches of SYDs. The contents of cinnamic acid showed the biggest differences $(0.17 \pm 0.08 \mathrm{mg} / \mathrm{g})$, followed by harpagoside $(0.42 \pm 0.19 \mathrm{mg} / \mathrm{g})$. We consider that it was probably due to the different origin of these crude herbs. It was reported that the contents of main constituents in Lonicerae japonicae flos, Scrophulariae radix, Angelicae sinensis radix, and Glycyrrhizae radix et rhizoma from different production areas differed greatly [24-27]. In order to ensure the efficiency of SYD, the crude drugs composed of SYD should be chosen from suitable production areas. In addition, the extraction temperature or time in the decocting process might also influence the content of each component in SYD. Further studies will be carried out to investigate the quality of each herb in SYD formula from different cultivation areas, and the decocting conditions of SYD formula, as well as the correlation between chromatographic fingerprints of chemical constituents and quality, so as to establish a comprehensive quality control system of SYD.

\section{Conclusion}

In the present study, an HPLC-DAD method was established and successfully applied for the simultaneous quantification of 13 major components in SYD for the first time to our knowledge. Thirteen marker compounds which have certain pharmacological activities were derived from the four individual herbs in SYD and could fully reflect the quality of the prescription. Validation of the method showed good linearity, repeatability, intra- and inter-day precision, and accuracy. The established method laid a good foundation for the quality control of SYD, while an effective and systematic quality control method for SYD still has a long way to go and requires further investigations.

\section{Conflict of Interest}

The authors declare that there is no conflict of interests regarding the publication of this paper.

Acknowledgments. The authors acknowledge Shineway Pharmaceutical Group Co., Ltd. for their financial support.

Table 4. Contents of 13 components in six batches of SYD

\begin{tabular}{|c|c|c|c|c|c|c|c|}
\hline \multirow[t]{2}{*}{ Compounds } & \multicolumn{7}{|c|}{ Contents (mg/g) } \\
\hline & Batch 1 & Batch 2 & Batch 3 & Batch 4 & Batch 5 & Batch 6 & $\overline{\text { Mean } \pm \mathrm{SD}}$ \\
\hline Harpagide & 2.50 & 1.26 & 1.57 & 1.43 & 1.62 & 2.43 & $1.80 \pm 0.53$ \\
\hline Neochlorogenic acid & 0.43 & 0.58 & 0.53 & 0.49 & 0.46 & 0.55 & $0.51 \pm 0.06$ \\
\hline Chlorogenic acid & 8.46 & 7.40 & 8.27 & 10.90 & 7.20 & 10.10 & $8.72 \pm 1.48$ \\
\hline Cryptochlorogenic acid & 0.93 & 1.07 & 0.62 & 1.15 & 0.91 & 1.13 & $0.97 \pm 0.20$ \\
\hline Ferulic acid & 0.36 & 0.21 & 0.25 & 0.44 & 0.24 & 0.24 & $0.29 \pm 0.09$ \\
\hline Isochlorogenic acid B & 0.67 & 0.83 & 0.71 & 0.75 & 0.69 & 0.82 & $0.75 \pm 0.07$ \\
\hline Isochlorogenic acid A & 1.57 & 1.38 & 1.29 & 1.82 & 1.33 & 1.99 & $1.56 \pm 0.28$ \\
\hline Isochlorogenic acid C & 1.26 & 1.45 & 1.33 & 1.53 & 1.25 & 1.56 & $1.40 \pm 0.14$ \\
\hline Angoroside C & 0.66 & 0.40 & 0.33 & 0.67 & 0.48 & 0.76 & $0.55 \pm 0.17$ \\
\hline Harpagoside & 0.76 & 0.33 & 0.28 & 0.43 & 0.48 & 0.26 & $0.42 \pm 0.19$ \\
\hline Cinnamic acid & 0.24 & 0.14 & 0.10 & 0.29 & 0.17 & 0.09 & $0.17 \pm 0.08$ \\
\hline Glycyrrhizic acid & 7.62 & 3.46 & 3.23 & 4.31 & 4.81 & 6.41 & $4.97 \pm 1.73$ \\
\hline Ligustilide & 0.17 & 0.06 & 0.07 & 0.09 & 0.10 & 0.14 & $0.10 \pm 0.04$ \\
\hline
\end{tabular}




\section{References}

1. Liu, Y.; Chi, S.; Wang, W.; Su, L.; Liu, B. Molecules 2017, 22, 1-16. 2. Liu, Y. F.; Tu, S. H.; Chen, Z.; Wang, Y.; Hu, Y. H.; Dong, H. Evid. Based Complement. Alternat. Med. 2014, 2014, 1-7.

3. Wang, Y. N.; Liu, H.; Zhang, J. S.; Ma, W. G.; Lu, Y.; Meng, F. X. Integr. Med. Res. 2015, 4, 57-58.

4. Li, N.; Qu, X.; Lin, S.; Li, J.; Li, H.; Lin, H.; Lv, G.; Tian, W.; Li, G.; Lin, Z. J. Jilin Univ. 2013, 39, 264-267.

5. Xu, Y.; Zhang, J.; Li, M.; Li, L.; Peng, L.; Zhang, G.; Yang, C.; Zhou, Y. Chin. J. Basic Med. Tradit. Chin. Med. 2012, 18, 161-163.

6. Zhao, Y.; Liu, M.; Zhang, Y.; Wang, B.; Zhang, Y.; Hao, Q.; Wei, J. Chin. J. Integr. Tradit. West. Med. 2017, 37, 79-85.

7. Peng, G.; Guan, H.; Wang, X.; Shi, Y. Acta Pharm. Sin. B 2017, 7, 193-201.

8. Guo, S.; Cui, X.; Jiang, M.; Bai, L.; Tian, X.; Guo, T.; Liu, Q.; Zhang,

L.; Ho, C.; Bai, N. J. Food Drug Anal. 2017, 25, 417-424.

9. Liu, Q.; Qin, K.; Shen, B.; Cai, H.; Cai, B. Acta Chromatogr. 2015, 27, 697-709.

10. Chi, S.; Wang, W.; Liu, B. J. Beijing Univ. Tradit. Chin. Med. 2016, 39, 933-940.

11. Shang, X.; Pan, H.; Li, M.; Miao, X.; Ding, H. J. Ethnopharmacol. 2011, 138, 1-21.

12. Wang, X. Q.; Wei, F. Y.; Wei, Z. F.; Zhang, L.; Luo, M.; Zhang, Y. H.; Zu, Y. G.; Fu, Y. J. Sep. Purif. Technol. 2014, 135, 80-87.

13. Xiong, J.; Li, S.; Wang, W.; Hong, Y.; Tang, K.; Luo, Q. Food Chem. 2013, 138, 327-333.
14. Seo, O. N.; Kim, G. S.; Park, S.; Lee, J. H.; Kim, Y. H.; Lee, W. S.; Lee, S. J.; Chi, Y. K.; Jin, J. S.; Sang, K. C. Food Chem. 2012, 134, 572-577. 15. Chen, Y. H.; Qi, J.; Hua, J.; Yu, B. Y. Chin. J. Nat. Med. 2014, 12, $47-54$

16. Jing, J.; Chan, C. O.; Xu, L.; Jin, D.; Cao, X.; Mok, D. K.; Parekh, H. S.; Chen, S. J. Pharm. Biomed. Anal. 2011, 56, 830-835.

17. Li, J.; Huang, X. Y.; Du, X. J.; Sun, W. J.; Zhang, Y. M. Nat. Prod. Res. 2009, 23, 775-780.

18. Wei, W. L.; Zeng, R.; Gu, C. M.; Qu, Y.; Huang, L. F. J. Ethnopharmacol. 2016, 190, 116-141.

19. Li, J.; Hua, Y.; Ji, P.; Yao, W.; Zhao, H.; Zhong, L.; Wei, Y. Pharm. Biol. 2016, 54, 1881-1890.

20. Deng, S.; Chen, S. N.; Yao, P.; Nikolic, D.; van Breemen, R. B.; Bolton, J. L.; Fong, H. H.; Farnsworth, N. R.; Pauli, G. F. J. Nat. Prod. 2006, 69, 536-541.

21. Zhang, Q.; Ye, M. J. Chromatogr. A 2009, 1216, 1954-1969.

22. Long, J ; Liang, B; Li, S.; Chen, Z J Sep. Sci. 2017, 40, 4847-4856.

23. China Pharmacopoeia Committee. Pharmacopoeia of the People's Republic of China (IV), China, Medical Science Press, Beijing 2015, pp. 374-377.

24. Qian, Z. M.; Li, H. J.; Li, P.; Ren, M. T.; Tang, D. Chem. Pharm. Bull. 2007, 55, 1073-1076.

25. Zhang, Y.; Cao, G.; Ji, J.; Cong, X.; Wang, S.; Cai, B. J. Sep. Sci. 2011, 34, 1429-1436.

26. Peng, Z.; Bi, Z. M.; Li, P.; Qi, L. W.; Yi, L.; Tang, D. Chromatographia 2008, 67, 973-978.

27. Wei, S. S.; Yang, M.; Chen, X.; Wang, Q. R.; Cui, Y. J. Chin. J. Nat. Med. 2015, 13, 232-240. 